\title{
Impact of round edge on the duct fluid flow: analytical investigation
}

\author{
Mehran Khaki Jamei ${ }^{1} \cdot$ Mohsen Heydari Alashti $^{1} \cdot$ Morteza Abbasi $^{1} \cdot$ Aliosat EbrahimNejad $^{1}$. \\ Mohammad Hasan Taheri ${ }^{1}$
}

Received: 10 July 2020 / Accepted: 24 February 2021 / Published online: 6 March 2021

(C) The Author(s) 2021 OPEN

\begin{abstract}
The present study aims to investigate the effect of the round edge on the laminar Newtonian fluid that flows through a channel. As an innovation, the sine and cosine transform functions are employed to solve the momentum governing equation in Cartesian and Cylindrical coordinates. Owing to the duct symmetric, only the quarter of the cross-section $(\theta=0$ to $\pi / 2)$ is analyzed. The analytical correlations for velocity distribution in both coordinates are provided; afterward, the effect of the round edge on the velocity profile has been investigated. It can be concluded that if a circular crosssection is replaced with a non-circular cross-section, the velocity profile becomes more uniform and less velocity variation is observed. Further, with a constant pressure gradient, among rectangular, round edge and circular cross-sections, the maximum velocity in a circular cross-section becomes minimum. In addition, it is observed that for the same pressure difference, an increase of $m$ value leads to the higher average velocity and mass flow.
\end{abstract}

Keywords Sine transform · Cosine transform · Super-ellipse function · Duct · Round edge

\section{Introduction}

Depending upon the shape and cross-section of the duct, the fluid has various velocity distribution. Ducts with different shapes such as rectangular, circular, trapezoidal and triangular are commonly used in several applications particularly heat exchangers (such as trapezoidal crosssection [1, 2], circular cross-section [3], and elliptic crosssection [4]), cooling systems (like microscale cooling [5], vortex cooling [6], and circular cooling [7]) and microchannels (for example arbitrary cross-section [8, 9], trapezoidal cross-section [10, 11], circular cross-section [12, 13]).

Plenty of studies on the different shape of the duct cross-section have been conducted during the decades. Yuan et al. [14] simulated fully developed laminar flow and heat transfer in fuel cell, numerically. They considered rectangular and trapezoidal cross-section. They applied finite volume method (FVM) for solving the problem. They reported that the aspect ratio has significant effect on the thermal-hydrualic parameters.

The efficiency dependency of the coolant on the channel's cross-section in microchannels is provided by Sharma et al. [15]. They analyzed the performance of trapezoidal (with two configurations $\mathrm{A}$ and $\mathrm{V}$-type) and rectangular microchannels. They modeled and solved the problem by FVM using FLUENT. They concluded that for the same flow rate for all geometries, one type of trapezoidal configuration offers an appropriate choice.

Zhang [16] provided an investigation on transient heat and mass transfer in a hexagonal cross-section duct. He investigated the problem both numerically and experimentally. He observed that the thickness of wall has notable effects unlike steady-state heat transfer tube.

Ciofalo and Di Liberto [17] employed Computational Fluid Dynamics (CFD) to investigate heat transfer in serpentine pipes for laminar and fully developed flow. They picked various curvature and curvature radius for each

Mehran Khaki Jamei, Mehran.khaki@gmail.com | 'Department of Mechanical Engineering, Sari Branch, Islamic Azad University, Sari, Iran. 
geometry. The numerical results were compared with experimental results.

Navardi et al. [18] yielded some correlations in a noncircular cross-section for velocity profiles and flow resistance. They discussed how to drive an analytical solution describing the flow profile for geometries like triangles or trapeziums or rectangles.

Letelier et al. [19] solved a steady laminar viscoelastic fluid flow in a straight tube with arbitrary cross-section. They used the mapping method to model the crosssection geometry. They applied the asymptotic series for expanding the variables. The temperature distributions for each cross-section were provided.

Heat transfer and flow characteristics of heat exchangers with different trapezoidal ducts was examined by Zhang et al. [20]. They observed that the temperature difference distribution and pressure drop in trapezoidal ducts are better than that of in rectangular ducts. Thus, the heat transfer enhances.

Moorthi and Sharma [21] examined the fully developed laminar fluid flow and heat transfer in non-circular subchannel geometries. The aforementioned geometries are representing a typical nuclear fuel bundle. They simulated the problem numerically in COMSOL multi physics code as classical PDEs. The effect of different aspect ratio on $f R e$, flow and temperature distributions. Moreover, correlations are provided to estimate flow and heat transfer characteristics as a function of $p / d$ ratio for non-circular sub-channels by least square regression analysis.

Here we motivated from the authors' previous work [22], and aim to apply both sine and cosine transform functions to investigate the influence of round edge on the fluid flow through a duct. Furthermore, the velocity distribution correlation in both Cartesian and Cylindrical coordinates will be obtained.

In the following sections, the problem is described, the governing equations and boundary conditions are defined, then using the dimensionless parameters, the equations are transformed into dimensionless form. Afterward, the solution methodology is defined and applied, then the validation of the results is presented, and in the next, the results and discussions are provided.

\section{Description of problem}

In this paper, we consider a fully developed, laminar, incompressible, Newtonian fluid flow in a ducts. We are going to investigating the impact of the duct edge on the flow. Because of the simplicity of the super-ellipse function toward other similar functions like hyper-ellipsoid function, it is preferred for modeling the duct edge. Generally, super-ellipse in Cartesian coordinates is defined as [23,24]:
$\left|\frac{x}{A}\right|^{m}+\left|\frac{y}{B}\right|^{m}=1$

where $m, A$ and $B$ are positive numbers. Replacing $x=r \cos \theta$ and $y=r \sin \theta$, the super-ellipse function can be represented in cylindrical coordinates as:

$\frac{1}{A^{m}}\left((r \cos \theta)^{m}+\frac{(r \sin \theta)^{m}}{\varepsilon^{m}}\right)=1$

where $\varepsilon=\frac{B}{A}$. Therefore, the radius of the cross-section, according to $\theta$ can be obtained as:

$r=\frac{A}{\left((\cos \theta)^{m}+\left(\frac{\sin \theta}{\varepsilon}\right)^{m}\right)^{\frac{1}{m}}}$

In the present research, we have chosen different $m$ to produce different edges as illustrated in Figs. 1 and 2.

\section{Governing equation}

Considering aforementioned assumption, the momentum equation is introduced as:

\subsection{Cartesian coordinate}

$\frac{1}{\mu} \frac{\partial P}{\partial x^{*}}=\left(\frac{\partial^{2} u^{*}}{\partial y^{* 2}}+\frac{\partial^{2} u^{*}}{\partial z^{* 2}}\right)$

\subsection{Cylindrical coordinate}

$\frac{1}{\mu} \frac{\partial P}{\partial z^{*}}=\left(\frac{1}{r^{*}} \frac{\partial v_{z}^{*}}{\partial r^{*}}+\frac{\partial^{2} v_{z}^{*}}{\partial r^{* 2}}+\frac{1}{r^{* 2}} \frac{\partial^{2} v_{z}^{*}}{\partial \theta^{2}}\right)$

Introducing the dimensionless parameters as:

$x=\frac{x^{*}}{h}, y=\frac{y^{*}}{h}, r=\frac{r^{*}}{R}, u(y, z)=\frac{\mu u^{*}}{h^{2}\left(-\frac{d P}{d x^{*}}\right)}, u(r, \theta)=\frac{\mu v_{z}^{*}}{R^{2}\left(-\frac{d P}{d z^{*}}\right)}$

Equations 4- 6 can be transformed into the dimensionless form, respectively, as:

$\frac{\partial^{2}}{\partial y^{2}} u(y, z)+\frac{\partial^{2}}{\partial z^{2}} u(y, z)=-1$

$\frac{\partial^{2}}{\partial r^{2}} u(r, \theta)+\frac{1}{r} \frac{\partial}{\partial r} u(r, \theta)+\frac{1}{r^{2}} \frac{\partial^{2}}{\partial \theta^{2}} u(r, \theta)=-1$

In addition, we consider the no-slip condition on the walls as boundary conditions. 


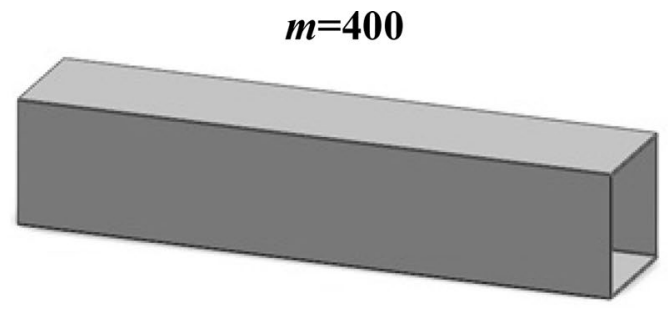

$m=4$

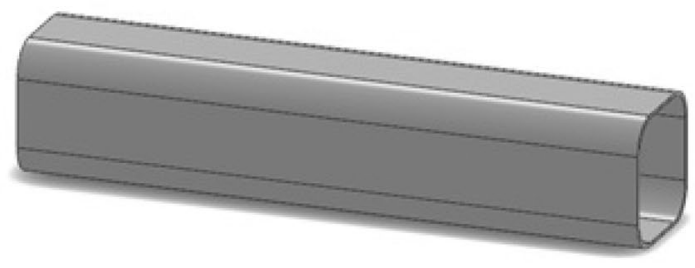

Fig. 1 Ducts produced with different $m$



Fig. 2 Edges produced with different $m$ value

\section{Methodology}

\subsection{Sine and cosine transform functions}

The momentum equation in both coordinates is solved analytically with the aid of sine and cosine transform functions. These functions help us to convert the partial differential equation (PDE) form into the ordinary differential equation (ODE) form; hence, the solving becomes convenient. In the following both of them are demonstrated.



$m=2$

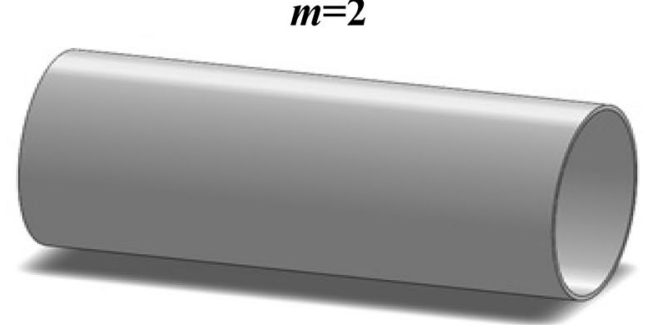

\subsubsection{Applying sine transform}

Using the sine transform function $[25,26]$ on Eq. (7), we have:

$S_{n}\left(\frac{\partial^{2}}{\partial y^{2}} u(y, z)+\frac{\partial^{2}}{\partial z^{2}} u(y, z)\right)=S_{n}(-1)$

$S_{n}\left[\frac{\partial^{2}}{\partial y^{2}} u(y, z)\right]=-\left(\frac{n \pi}{2 d}\right)^{2} S_{n}(z)+\left(\frac{2 n \pi}{2 d}\right)\left[u(0, z)+(-1)^{n} u(2 h, z)\right]$

$S_{n}\left[\frac{\partial^{2}}{\partial z^{2}} u(y, z)\right]=S_{n}^{\varepsilon}(z)$

$S_{n}[-1]=-\left(\frac{2}{2 d}\left(\frac{\left(1-(-1)^{n}\right)}{n \pi / 2 d}\right)\right)$

Considering the non-slip boundary condition, we can write:

$S_{n}\left[\frac{\partial^{2}}{\partial y^{2}} u(y, z)\right]=-\left(\frac{n \pi}{2 d}\right)^{2} S_{n}(z)$

Consequently, Eqs. (9) and (10) reduce to:

$S_{n}^{\prime \prime}(Z)-\left(\frac{n \pi}{2 d}\right)^{2} S_{n}(Z)=-\frac{2\left(1-(-1)^{n}\right)}{n \pi}, n=1,3,5, \ldots$

By solving Eq. (11); subsequently, applying the invers sine transform, the dimensionless velocity distribution in Cartesian coordinate is obtained as follow: 
$u(y, z)=\sum_{n=1}^{\infty} S_{n}(Z) \cdot \operatorname{Sin}\left(\frac{n \pi y}{2}\right), n=1,3,5, \ldots$

\subsubsection{Applying cosine transform}

We can employ similar procedure for Eq. (8) for cosine transform function $[25,26]$; accordingly, we obtain:

$C_{n}\left(\frac{\partial^{2}}{\partial r^{2}} u(r, \theta)+\frac{1}{r} \frac{\partial}{\partial r} u(r, \theta)+\frac{1}{r^{2}} \frac{\partial^{2}}{\partial \theta^{2}} u(r, \theta)\right)=C_{n}(-1) ;$

$C_{n}\left[\frac{\partial^{2}}{\partial r^{2}} u(r, \theta)\right]=C_{n}^{\prime \prime}(r)$

$C_{n}\left[\frac{1}{r} \frac{\partial}{\partial r} u(r, \theta)\right]=\frac{1}{r} C_{n}^{\prime}(r)$

$C_{n}\left[\frac{1}{r^{2}} \frac{\partial^{2}}{\partial \theta^{2}} u(r, \theta)\right]=\frac{1}{r^{2}}\left[(4 n)^{2} C_{n}(r)-\frac{8}{\pi}\left(u_{\theta}(r .0)-(-1)^{n+1} u_{\theta}\left(r \cdot \frac{\pi}{2}\right)\right)\right]=\frac{(4 n)^{2}}{r^{2}} C_{n}(r)$
$C_{n}[-1]=\left\{\begin{array}{c}-1 n=0 \\ 0 n \neq 0\end{array}\right.$

Note that considering symmetry, we get:

$C_{0}^{\prime \prime}(r)+\frac{1}{r} C_{0}^{\prime}(r)+1=0$, for $n=0$

$C_{n}^{\prime \prime}(r)+\frac{1}{r} C_{n}^{\prime}(r)-\frac{(4 n)^{2}}{r^{2}} C_{n}(r)=0$, for $n=1,2,3, \ldots$

To obtain the dimensionless velocity distribution in cylindrical coordinate, Eq. (14) and (15) are solved, and we have:

$u(r, \theta)=-\frac{1}{4} r^{2}+A+2 \sum_{n=1}^{10} B_{n} r^{4 n} \cos (4 n \theta)$

where the values of $A$ and $B_{n}$ can be obtained using no-slip condition.

It is worth to note that due to using the cartesian coordinate for the rectangular cross-section, the no-slip condition can be applied (zero velocity on the walls). However, for non-circular cross-sections, the cylindrical coordinate and the symmetric condition at 0 and $\pi / 2$ angles have been used deliberately. In other words, the condition of velocity differentiation in respect to $\theta$ has been used at these two points. Also, it can be seen that for $m=400$ (big $m$ ), the results of sine transformation for cartesian and cosine transformation for cylindrical has become the same.

\section{Results and discussion}

In Fig. 3, the comparison of sine transforms results with other conventional analytical methods introduced in ref. [27] is depicted. It can be seen that the sine transform method results are in reasonable agreement with the analytical method.

In Fig. 4, we show 3D dimensionless velocity distributions for various $m$. Accordingly, the maximum velocity occurred at $r=0$, or at the center of the cross-section in all angles. Figure 5, depicts the impact of dimensionless velocity distribution contours of the whole cross-section. In this figure, since the cross-section is too small, the velocity distribution intensifies near the center.

The velocity distribution in the duct at different angles on the cylindrical coordinates by cosine transform function for various $m$ is illustrated in Fig. 6 . Further, the velocity distribution in the duct at different radius on by cosine transform function for various $m$ is presented in
Fig. 7. It can be seen when the value of $m$ decreases, the slope of the velocity profiles near the wall increases, the value velocity for different $\theta$ tends to be the same; hence, the velocity profiles overlap. Besides, as the value of $m$ reduces, the velocity profile in different $r$ becomes uniform, therefore, we have less fluctuation in the velocity distribution. It means that the round edge leads to more uniform velocity distribution.

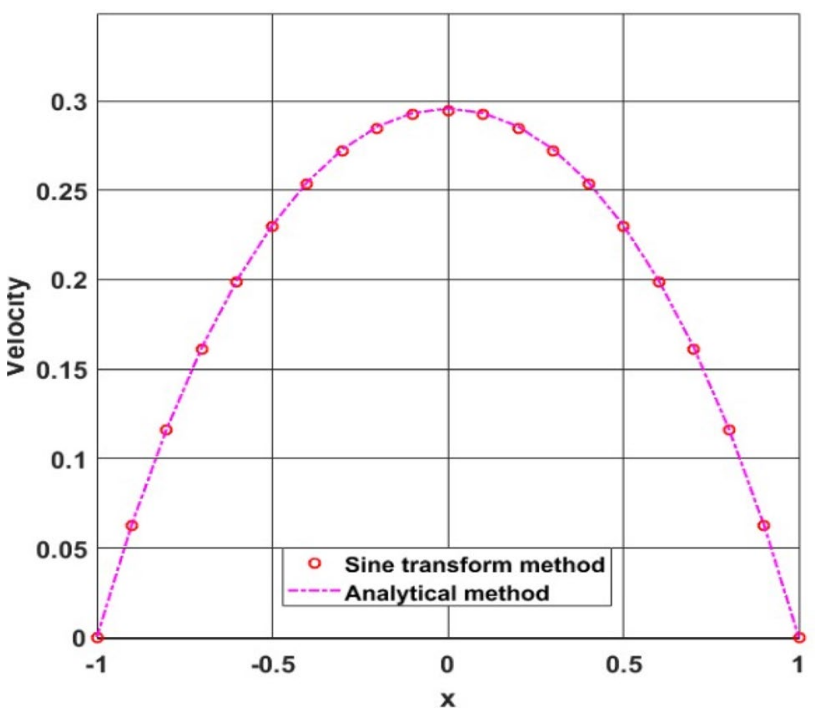

Fig. 3 Comparison of Sine transform method with analytical method 

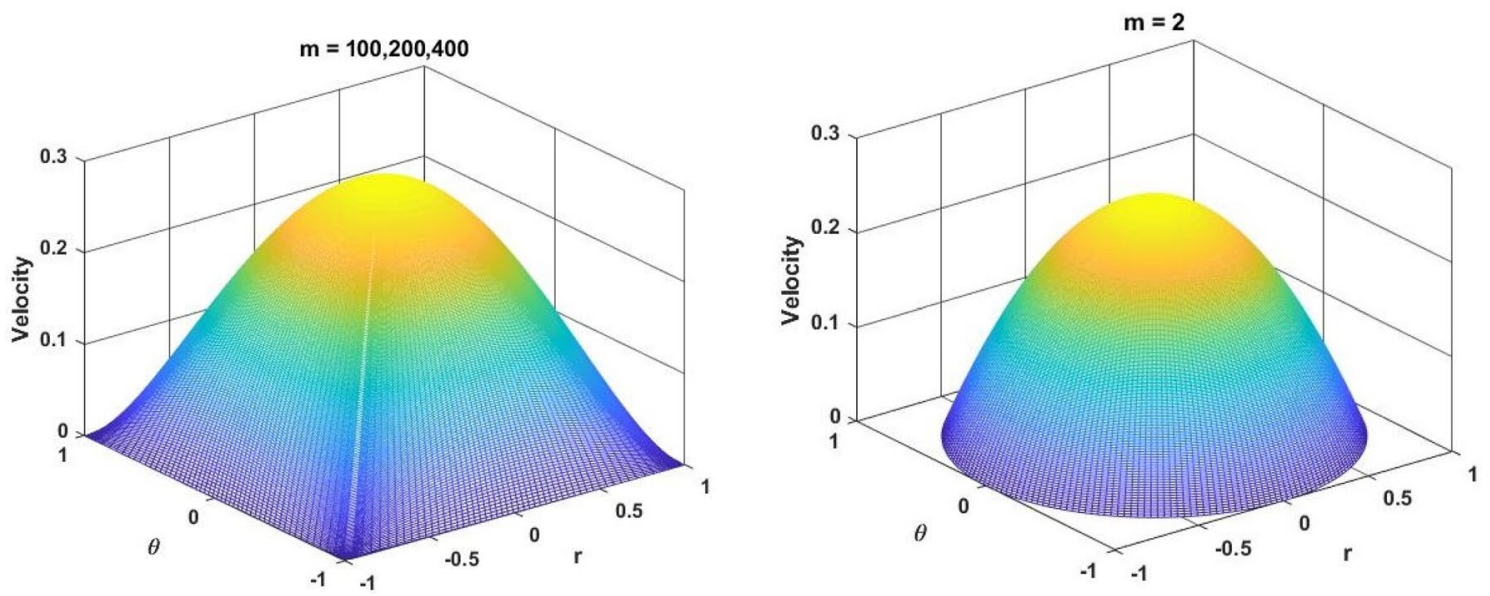

Fig. 4 3D velocity distribution for different $m$ value
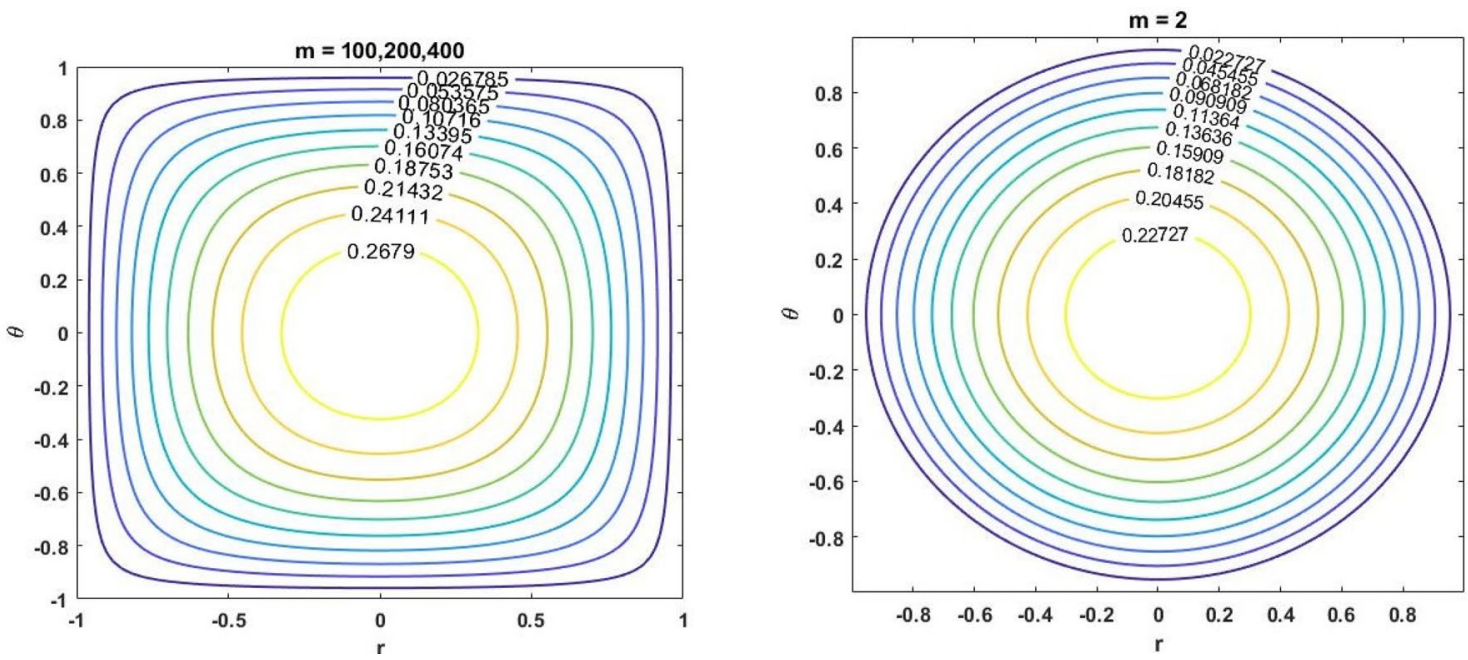

Fig. 5 Velocity contour for different $m$ value

Figure 8 shows the variation of flow velocity distributions for radius from 0 to 1 and various $m$ value. We can observe due to less effect of the duct wall on the flow, the velocity remains unchanged at the center of the cross section $(r=0)$ for all $m$ value. The value of the velocity differs, however for $m=400,200,100$ are almost equal.

Also, it can be seen that the velocity for $m=400,200$, 100 (rectangular cross section) has the highest value, but when the edge round becomes completely circular $(m=2)$, it becomes minimum. This can be interpreted that the wall shear stress of a circular cross-section is larger than a rectangular cross-section profile. It can be found that as the edge shape changed from the rectangular cross-section into the circular one, roundness of the cross-section influences the flow velocity, and the value of it becomes smaller.
The flow velocity distributions of the duct for various $m$ and angles from $\theta=0$ to $\pi / 2$ is demonstrated in Fig. 9. It is demonstrated for $\theta=0$ and $\pi / 2$, the velocity values at the wall $(r=1)$ are zero in all cases; however, at other angles and with a constant radius it is not zero. In that case, you move away from the wall, depending on the cross-section shape (Fig. 2); thus, the velocity becomes non-zero.

The minimum and maximum value of the velocity occur at $m=2$ and $m=400,200,100$, respectively for all $\theta$, and the maximum velocity values $(r=0)$ in each shape differ from another, due to differences in cross-section. Moreover, near the round corner $(\theta=\pi / 4)$, the velocity values increase more than other points, due to roundness effect of that point. Besides, it should be noted that for $m=2$ (circular shape), the velocity profile remains unchanged at different angles. 

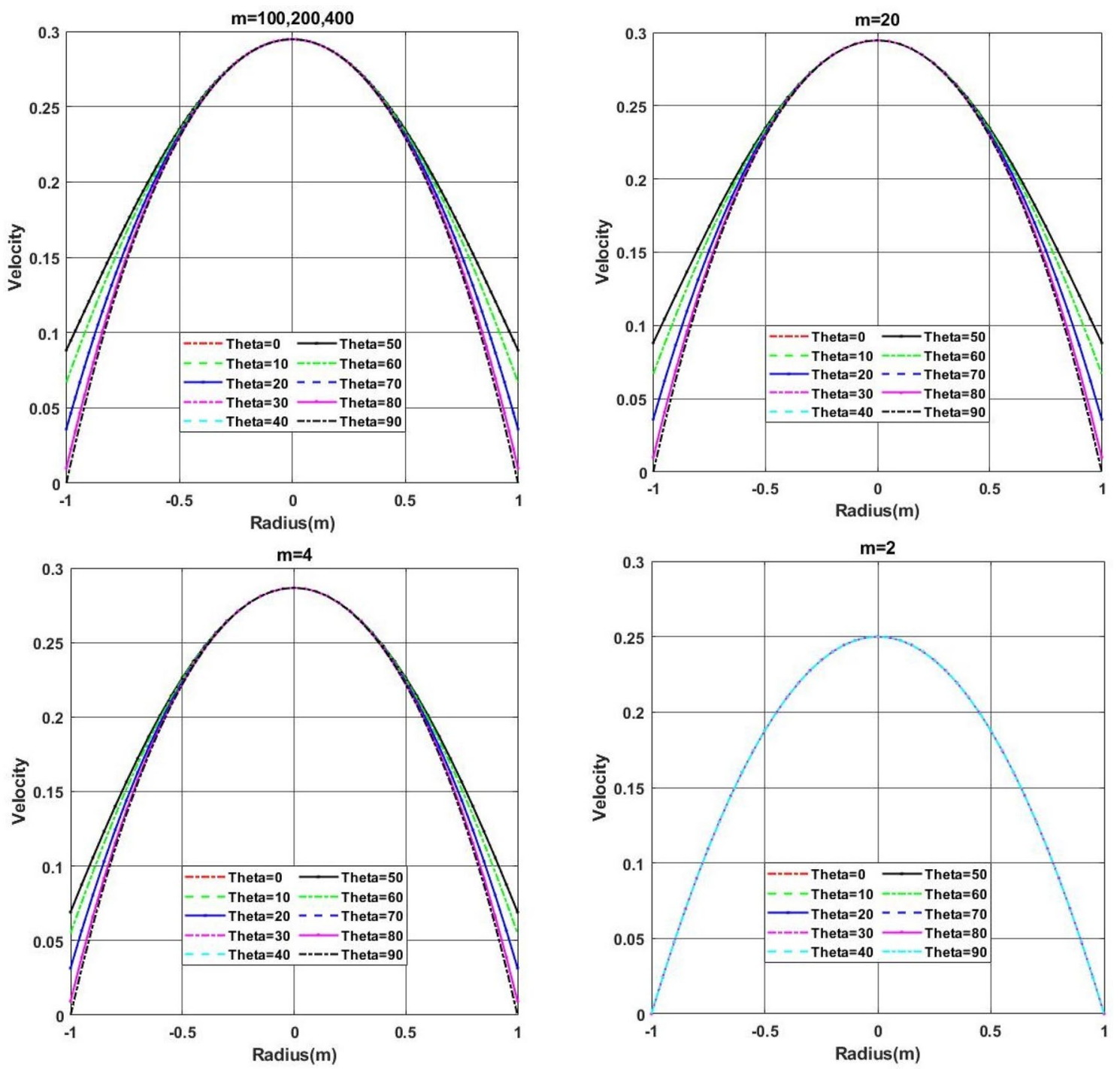

Fig. 6 velocity profile in the duct at different angles by cosine transform function for various $m$

The dimensionless value of the average velocity in different $\mathrm{m}$ is plotted in Figs. 10 and 11. When $m$ increases, the values of maximum velocity $\left(V_{\max }\right)$ and the average one $\left(V_{\text {av }}\right)$ is increased. However, in $m \geq 4$, the values of maximum velocity and the average velocity almost equal. Also, it can be seen that when the identical pressure difference is applied, by increasing $m$, the average velocity enhances; thus, the mass flow increases.

\section{Conclusion}

The purpose of this research is to investigate the effect of the round edge on the fluid flow through the duct. Using the super-ellipse function, the cross-section of the duct changes from rectangular into circular configuration. The sine and cosine transform function is applied to transform the momentum governing equation into 



Fig. 7 velocity profile in the duct at different angles by cosine transform function for various $m$

the ODE form and the analytical expression for the velocity distribution is obtained for both Cartesian and Cylindrical coordinates. The effect of round edge on the flow velocity profile was analyzed and discussed. It was revealed when the cross-section becomes circular, the velocity becomes more uniform toward the rectangular and round edge cross-section. Besides, it was concluded that at different angles of circular cross-section, the velocity profiles remain constant; whereas, for the round edge and rectangular cross-sections, velocity profile was changed. Furthermore, the maximum and minimum value of the velocity was observed in $m=2$ and 400 , respectively. 

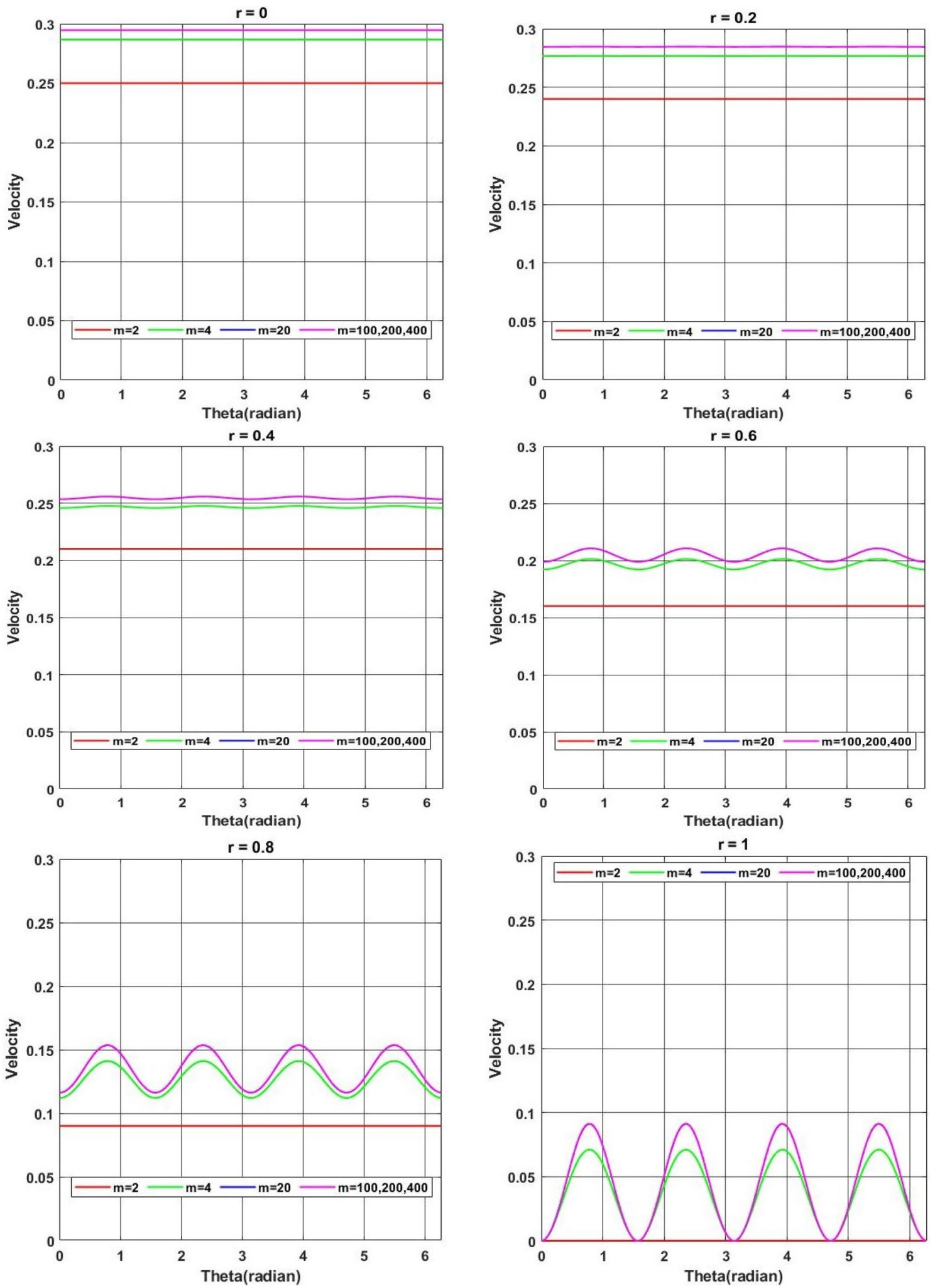

Fig. 8 Comparison of velocity profiles for different $r$ 



Fig. 9 Comparison of velocity profiles for different $\theta$ 

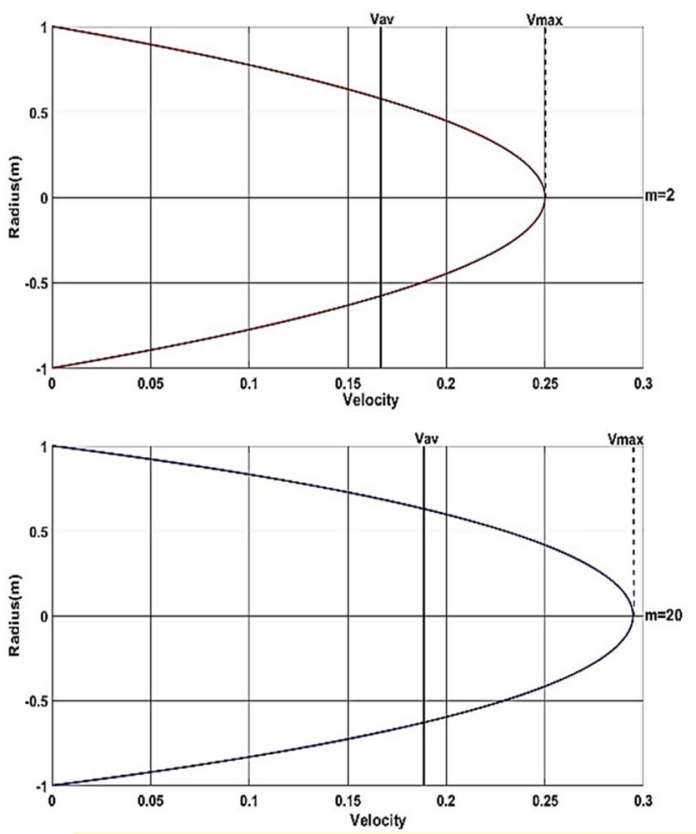
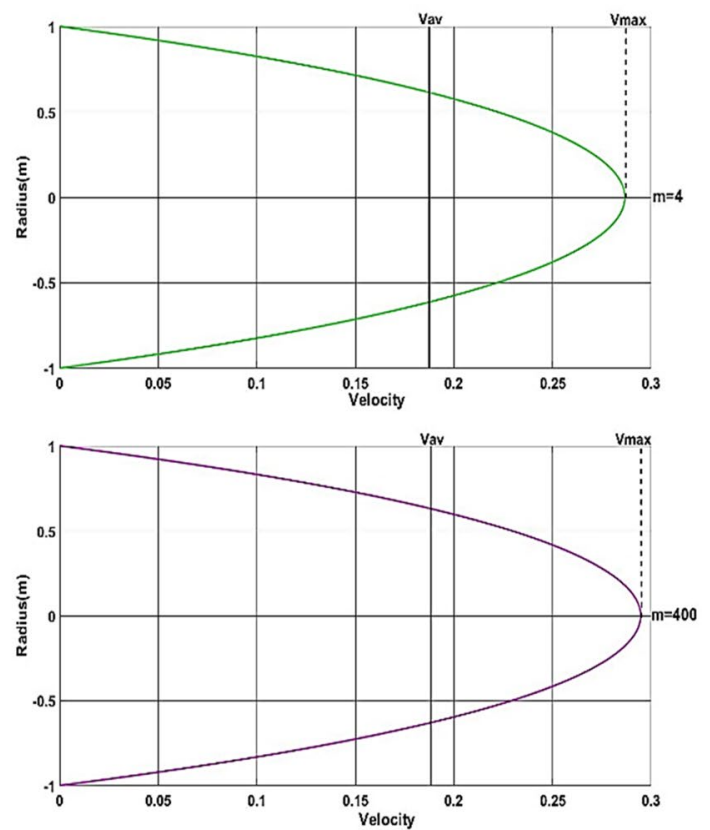

Fig. 10 comparison of maximum velocity $\left(V_{\max }\right)$ and average velocity $\left(V_{\text {av }}\right)$ in different $m$

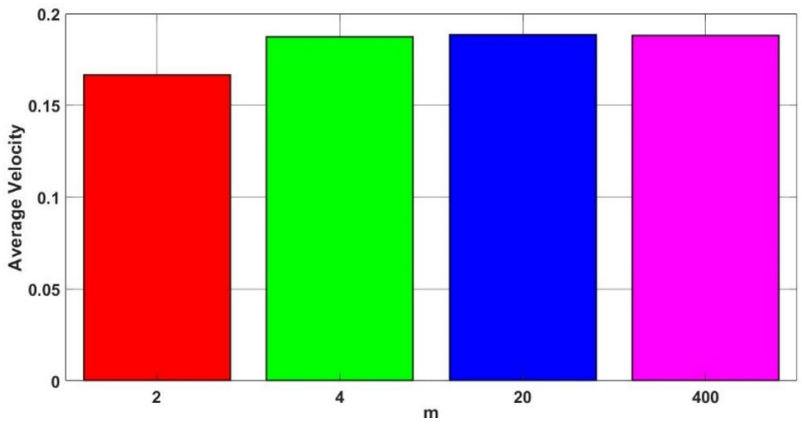

Fig. 11 Variation of average velocity $\left(V_{a v}\right)$ in various $m$

\section{Declaration}

Conflict of interest The authors declare that they have no conflict of interests.

Open Access This article is licensed under a Creative Commons Attribution 4.0 International License, which permits use, sharing, adaptation, distribution and reproduction in any medium or format, as long as you give appropriate credit to the original author(s) and the source, provide a link to the Creative Commons licence, and indicate if changes were made. The images or other third party material in this article are included in the article's Creative Commons licence, unless indicated otherwise in a credit line to the material. If material is not included in the article's Creative Commons licence and your intended use is not permitted by statutory regulation or exceeds the permitted use, you will need to obtain permission directly from the copyright holder. To view a copy of this licence, visit http://creativecommons .org/licenses/by/4.0/.

\section{References}

1. Esmaeilzadeh A, Amanifard N, Deylami HM (2017) Comparison of simple and curved trapezoidal longitudinal vortex generators for optimum flow characteristics and heat transfer augmentation in a heat exchanger. Appl Therm Eng 125:1414-1425

2. Abed AM, Alghoul MA, Sopian K, Mohammed HA, Majdi HS, Al-Shamani AN (2015) Design characteristics of corrugated trapezoidal plate heat exchangers using nanofluids. Chem Eng Process Process Intensif 87:88-103

3. Karimi A, Afrand M (2018) Numerical study on thermal performance of an air-cooled heat exchanger: effects of hybrid nanofluid, pipe arrangement and cross section. Energy Convers Manage 164:615-628

4. Dogan S, Darici S, Ozgoren M (2019) Numerical comparison of thermal and hydraulic performances for heat exchangers having circular and elliptic cross-section. Int J Heat Mass Transf 145:118731

5. Jiang XN, Zhou ZY, Huang XY, Liu CY. (1997) Laminar flow through microchannels used for microscale cooling systems. In: Proceedings of the 1st Electronic Packaging Technology Conference (Cat No97TH8307)1997. (p 119-22)

6. Wang J, Du C, Wu F, Li L, Fan X (2019) Investigation of the vortex cooling flow and heat transfer behavior in variable crosssection vortex chambers for gas turbine blade leading edge. Int Commun Heat Mass Transf 108:104301

7. You J, Feng H, Chen L, Xie Z, Xia S (2020) Constructal design and experimental validation of a non- uniform heat generating body with rectangular cross-section and parallel circular cooling channels. Int J Heat Mass Transf 148:119028

8. Huang P, Dong G, Zhong X, Pan M (2020) Numerical investigation of the fluid flow and heat transfer characteristics of treeshaped microchannel heat sink with variable cross-section. Chem Eng Process Process Intensif 147:107769 
9. Bahrami M, Yovanovich M, Culham J (2006) Pressure drop of fully-developed, laminar flow in microchannels of arbitrary cross-section. J Fluids Eng 128(5):1036-1044

10. Pan M, Zhong Y, Xu Y (2018) Numerical investigation of fluid flow and heat transfer in a plate microchannel heat exchanger with isosceles trapezoid-shaped reentrant cavities in the sidewall. Chem Eng Process Process Intensif 131:178-189

11. Behnampour A, Akbari OA, Safaei MR, Ghavami M, Marzban A, Sheikh Shabani GA et al (2017) Analysis of heat transfer and nanofluid fluid flow in microchannels with trapezoidal, rectangular and triangular shaped ribs. Physica E 91:15-31

12. Issa $L$ (2018) A simplified model for unsteady pressure driven flows in circular microchannels of variable cross-section. Appl Math Model 59:410-426

13. Effati E, Pourabbas B (2018) New portable microchannel molding system based on micro-wire molding, droplet formation studies in circular cross-section microchannel. Mater Today Commun 16:119-123

14. Yuan J, Rokni M, Sundén B (2001) Simulation of fully developed laminar heat and mass transfer in fuel cell ducts with different cross-sections. Int J Heat Mass Transf 44:4047-4058

15. Sharma D, Singh PP, Garg H (2013) Comparative study of rectangular and trapezoidal microchannels using water and liquid metal. Procedia Eng 51:791-796

16. Zhang L-Z (2015) Transient and conjugate heat and mass transfer in hexagonal ducts with adsorbent walls. Int J Heat Mass Transf 84:271-281

17. Ciofalo M, Di Liberto M (2015) Fully developed laminar flow and heat transfer in serpentine pipes. Int J Therm Sci 96:248-266

18. Navardi S, Bhattacharya S, Azese M (2016) Analytical expression for velocity profiles and flow resistance in channels with a general class of noncircular cross sections. J Eng Math 99:103-118

19. Letelier MF, Hinojosa CB, Siginer DA (2017) Analytical solution of the Graetz problem for non-linear viscoelastic fluids in tubes of arbitrary cross-section. Int J Therm Sci 111:369-378

20. Zhang X, Wang Y, Jia R, Wan R (2017) Investigation on heat transfer and flow characteristics of heat exchangers with different trapezoidal ducts. Int J Heat Mass Transf 110:863-872

21. Moorthi A, Sharma AK (2018) Laminar fluid flow and heat transfer in non-circular sub-channel geometries of nuclear fuel bundle. Prog Nucl Energy 103:243-253

22. Khaki M, Taeibi-Rahni M, Ganji D (2012) Analytical solution of electro-osmotic flow in rectangular nano-channels by combined Sine transform and MHPM. J Electrostat 70:451-456

23. Duchemin M, Tugui C, Collee V (2017) Optimization of contact profiles using super-ellipse. SAE Int J Mater Manuf 10:234-244

24. Burley IJ. (1985) Circular-to-rectangular transition ducts for highaspect ratio nonaxisymmetric nozzles. In: 21 st Joint Propulsion Conference (p 1346)

25. Britanak V, Yip PC, Rao KR (2007) CHAPTER 1 - discrete cosine and sine transforms. In: Britanak V, Yip PC, Rao KR (eds) Discrete cosine and sine transforms. Academic Press, Oxford, pp 1-15

26. Britanak V, Yip PC, Rao KR (2007) CHAPTER 5 - integer discrete cosine/sine transforms. In: Britanak V, Yip PC, Rao KR (eds) Discrete cosine and sine transforms. Academic Press, Oxford, pp 141-304

27. White FM (1991) Viscous fluid flow, 2nd edn. McGraw-Hill, New York

Publisher's Note Springer Nature remains neutral with regard to jurisdictional claims in published maps and institutional affiliations. 